\title{
A multi-agent architecture integrating learning and fuzzy techniques for landmark-based robot navigation
}

\author{
D. Busquets ${ }^{\dagger}$, R. López de Màntaras ${ }^{\dagger}$, C. Sierra ${ }^{\dagger}$, T. G. Dietterich ${ }^{\ddagger}$ \\ † Institut d'Investigació en Intel.ligència Artificial \\ Consell Superior d'Investigacions Científiques \\ Campus UAB, 08193 Bellaterra, Barcelona \\ \{didac,mantaras,sierra\}@iiia.csic.es \\ $\ddagger$ Oregon State University \\ Corvallis, OR, 97331 USA \\ tgd@cs.orst.edu
}

\begin{abstract}
This paper extends a navigation system implemented as a multi-agent system (MAS). The arbitration mechanism controlling the interactions between the agents was based on manually-tuned bidding functions. A difficulty with hand-tuning is that it is hard to handle situations involving complex tradeoffs. In this paper we explore the suitability of reinforcement learning for automatically tuning agents within a MAS to optimize a complex tradeoff, namely the camera use.
\end{abstract}

Keywords: machine learning, robotics, multiagent systems, fuzzy logic

\section{Introduction}

In landmark-based navigation, the robot must be able to start in an unknown location and navigate to a desired target using visually-acquired landmarks. The specific scenario that we are studying assumes that there is a target landmark that the robot is able to recognize visually. The target is visible from the robot's initial location, but it may subsequently be occluded by intervening objects. The challenge for the robot is to acquire enough information about the environment (locations of other landmarks and obstacles) so that it can move along a path from the starting location to the target position. The robot should do this quickly but safely.

In this paper, we build upon the multi-agent architecture for outdoor landmark-based navigation described in [14]. Each of the agents in the navigation system has a bidding function that is controlled by a set of internal parameters. These parameters need to be tuned to achieve the best performance of the Navigation system and of the overall system. Adjusting these parameters manually can be very difficult, particularly because of the tradeoffs confronting the top-level agents. An alternative to manual tuning is to employ machine learning techniques, specifically reinforcement learning (RL) methods. In this paper, we describe some experiments to test the feasibility of applying RL within this multi-agent system.

$\mathrm{RL}$ is most needed and most appropriate in cases where there is a complex, quantitative tradeoff between behaviors. In such cases, manual tuning is difficult, and the quantitative criterion of maximizing expected reward, which is the goal of RL, permits us to represent the tradeoff nicely. In the navigation system, such a tradeoff appears with the use of the camera, since several agents compete for using it. Moreover, its use is expensive, so we want to minimize it.

Section 2 is devoted to relevant related work. The multi-agent architecture of the navigation system is described in Section 3. Section 4 describes the learning task. The details of the RL algorithm we have used are explained in Section 5. The experiments are presented in Section 6. Finally, the paper concludes with Section 7.

\section{Related work}

Since Brooks proposed the subsumption architecture [4], many other coordination mechanisms for robotic systems have been proposed (Maes [10], [1]). Regarding multi-agent architectures, Liscano et al [7], Isik [8], and Stentz [15], among others, use hi- 
erarchical centralized architectures with arbitration to decide which activity takes control of the robot. Our approach, however, is completely decentralized, which means that the broadcast of information is not hierarchical. This approach is easier to program and is more flexible and extensible than centralized approaches. We propose a model for cooperation and competition between activities based on a simple bidding mechanism. A similar model was proposed by Rosenblatt [13] in CMU's DAMN project, in which voting was used to coordinate a set of modules to control the robot.

The map building approach we use is based on the work by Prescott [12], who proposed a network model that stores the spatial relationships among landmarks for robot navigation. By matching a perceived landmark with the network, the robot can find its way to a target, provided it is represented in the network. While Prescott's approach is quantitative, ours uses a fuzzy extension of his model to work with fuzzy qualitative information about distances and directions. Levitt and Lawton [9] also proposed a qualitative approach to the navigation problem, but assume unrealistically accurate distance and direction information between the robot and the landmarks. Another qualitative method for robot navigation was proposed by Escrig and Toledo [6], using constraint logic. However, they assume the robot has some a priori knowledge of the spatial relationship of the landmarks, while we build these relationships while exploring the environment.

\section{The multiagent architecture}

The architecture is composed of three systems (see Figure 1). Each system competes for two available resources: motion and vision. The three systems have the following responsibilities. The Pilot is responsible for all motions of the robot. It selects these motions to carry out commands from the Navigation system and (independently) to avoid obstacles. The Vision system is responsible for identifying and tracking landmarks (including the goal) and for detecting obstacles to support obstacle avoidance. The process of identifying landmarks requires comparing images taken before and after suitable robot motions, so the Vision system must ask the Pilot system to carry out certain motions to support perception. Finally, the Navigation system is responsible for choosing higher-level robot motions to move the robot to a specified goal. This requires requesting the Vision system to identify and track landmarks, to build a map of the environment, and requesting the Pilot to move the robot in various directions (to aid the Vision system or to move the robot toward the goal position or toward some intermediate target position).

From this brief description, two observations can be made. First, these three systems must cooperate to achieve the overall task of reaching the goal landmark position. For instance, the Pilot needs the Vision system to identify obstacles, and it needs the Navigation system to select a path to the goal. Second, the systems are also competing-there are some tradeoffs between them. For example, the Pilot and the Navigation system both compete for the Vision system. The Pilot needs vision for obstacle avoidance, while the Navigation system needs vision for landmark detection and tracking.

To manage this cooperation and competition, we use a bidding mechanism. Each system generates bids for the services offered by the Pilot and Vision systems. The service actually executed by each system depends on the winning bid at each point in time. We have manually written the bidding functions to obtain good performance from the combined system.

The Navigation system itself is also implemented as a MAS (see Figure 1). This system is composed of six agents with the following responsibilities: keep the target located with maximum precision and reach it (Target Tracker), keep the risk of losing the target low (Risk Manager), recover from blocked situations (Rescuer), keep the error in the distance to landmarks low (Distance Estimator), and keep the information on the map consistent and up-to-date (Map Manager). There is an additional agent, Communicator, which manages the communication between the Navigation system with the robot's other systems. As with the overall system, the Navigation system employs a bidding mechanism to coordinate these agents. Each agent bids for the action it wants the robot to perform. These bids are sent to the Communicator agent, which determines the winning action. The selected action is then sent as the Navigation system's bid for the services of the Vision and Pilot systems. Each action can involve a combination of requests to the Vision and the Pilot systems.

For map representation and wayfinding, we extended Prescott's beta-coefficients system. Prescott's model stores the relationships among 

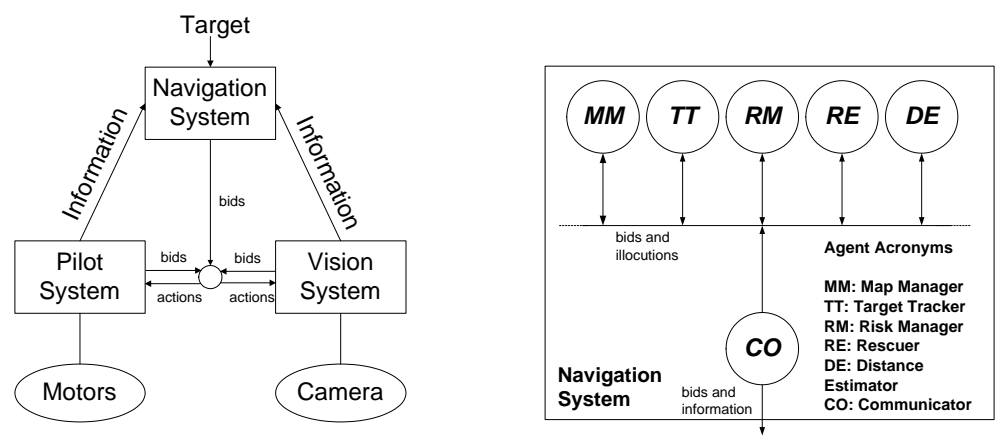

Figure 1: Left: Robot architecture. Right: Navigation System.

the landmarks in the environment to build a map. The location of a landmark is encoded based on the relative locations (headings and distances) of three other landmarks. This relationship is unique and invariant to viewpoint. Once this relationship has been stored, the location of each landmark can be computed from the locations of the three landmarks encoding it, no matter where the robot is located as long as the robot can compute the heading and distance to each of the three landmarks.

As the robot explores the environment, it stores the relationships among the landmarks it sees. This creates a network of relationships among the landmarks in the environment. If this network is sufficiently-richly connected, then it provides a computational map of the environment. Given the headings and distances to a subset of currentlyvisible landmarks, the network allows to compute the locations of all of the remaining landmarks, even if they are currently not visible from the robot.

If there are landmarks associated with all known obstacles in the environment, then this network can also be employed to plan a path from the current location of the robot to the goal. Because of obstacles, this path may not be a straight line from the current location to the goal. Instead, it typically consists of a sequence of intermediate targets, called diverting targets, such that if the robot travels from one diverting target to the next, it will eventually reach the goal.

Prescott's model assumes that the robot is able to measure the exact location of the landmarks. But this is not the case in our robot: the vision system gives only imprecise information about the location of the landmarks, and we cannot rely on the odometry of the robot, as it is also imprecise. To deal with this unavoidable imprecision, our extended model represents all the network coordinates as fuzzy numbers and carries out all map computations using fuzzy arithmetic [3].

\section{The learning task}

Within the Navigation system, a tradeoff exists between the Target Tracker agent, the Risk Manager, and the Distance Estimator. For instance, the Target Tracker wants to know the exact heading and distance to the target at all times, while the Risk Manager wants to ensure that the robot is surrounded by a rich network of landmarks so that the robot does not get lost. These agents' goals compete for the control of the camera. In addition to this conflict between these agents, the Navigation system also must not monopolize the camera, because the Pilot needs to use the camera for obstacle avoidance as well.

We propose to replace the Target Tracker, the Risk Manager, and the Distance Estimator with a new Learning Agent whose bidding function will be tuned by RL. We formulate the reward function for this agent so that it is rewarded for reaching the current target while minimizing the use of the camera. The two remaining agents have very different roles. The Map Manager maintains the betacoefficient map, but does not bid on actions. The only remaining active agent is the Rescuer, which is responsible for the higher-level choice of diverting targets whenever the robot becomes blocked. This activity is better-implemented by path planning algorithms than by RL, so we have not included the Rescuer's responsibilities within the Learning Agent.

The task confronting the Learning Agent is to choose actions (for both motion and vision) to reach 
the current target while minimizing the use of the camera. The current target is determined by the Rescuer, and the Map Manager provides the map information needed by the Learning Agent. If the robot becomes blocked, the Rescuer will choose a diverting target, and then the Learning Agent will take control and choose actions to reach that new target. Once the diverting target is reached, the Rescuer may be able to set the current target to be the original goal, and then the Learning Agent will attempt to move to that target.

\section{The RL algorithm}

There are two general styles of RL algorithms: Model-based and Model-free. Model-based algorithms learn a transition model $P\left(s^{\prime} \mid s, a\right)$ for the environment, where $s$ is the state of the environment at time $t, a$ is an action to be executed, and $s^{\prime}$ is the resulting state of the environment at time $t+1$. Model-based algorithms also learn a reward model $R\left(s, a, s^{\prime}\right)$ which gives the expected one-step reward of performing action $a$ in state $s$ and making a transition to state $s^{\prime}$.

For robot learning model-free methods are impractical, because they require many more interactions with the environment to obtain good results.

Hence, for our experiments, we have chosen the model-based algorithm known as Prioritized Sweeping [11].

We represent both the transition model $P\left(s^{\prime} \mid s, a\right)$ and the reward model $R\left(s, a, s^{\prime}\right)$ by threedimensional matrices with one cell for each combination of $s, s^{\prime}$, and $a$. This technique will only work if the state and action spaces are small. Hence, the most challenging aspect of applying $R L$ is the proper design of the state representation.

\subsection{State Representation}

We want the Learning Agent to learn a general policy that works for any environment, independent of the locations of the landmarks and targets. Hence, our state representation must not directly employ the locations of the landmarks. Moreover, the robot cannot directly observe the complete state of the environment, which would include the location of the robot, all obstacles, and all landmarks! Instead, the task of the robot is to learn under conditions of incomplete knowledge about the locations of obstacles, landmarks, and targets.
State spaces that encode incomplete knowledge are known as "belief state spaces" [5]. The purpose of a belief state representation is to capture the current state of knowledge of the agent, rather than the current state of the external world. We do not use the term belief state in the restricted sense of a probability distribution over states; the main reason to use a belief state representation is that it allows us to treat the states as observable. In our case, the learning agent is trying to move from a starting belief state in which it knows nothing to a goal belief state in which it is confident that it is located at the target state. Along the way, it seeks to avoid getting lost (a belief state in which it does not know its location relative to the target).

To explain our state representation, we begin by defining a set of belief state variables. Then we explain how these are discretized to provide a small set of features each taking on a small set of values so that $P\left(s^{\prime} \mid s, a\right)$ and $R\left(s, a, s^{\prime}\right)$ can be represented with small tables.

At any given point in time, the headings to all objects (landmarks and the target position) are divided into six sectors of 60 degrees each. The field of view of the robot is 60 degrees, so at any point in time, the robot can observe one sector. For each sector, we represent information about the number of landmarks believed to be in that sector and the precision of our beliefs about their headings and distances.

Given these sectors, the following state variables can be defined:

- Distance to target, and its imprecision, $D(t), I_{d}(t)$

- Heading to target, and its imprecision, $H(t), I_{h}(t)$

- The landmarks in each sector, $L(s)=$ $\left\{l_{1}, \ldots, l_{n_{s}}\right\}$

- Number of landmarks in each sector, $N(s)=$ $\min (4,|L(s)|)$

- Average imprecision of landmarks in each sector,

$$
\bar{I}(s)=\frac{1}{N(s)} \sum_{l \in B e s t(4, L(s))} I(l)
$$

We now explain each of these. The distance $D(l)$ to a landmark (or $D(t)$ to the target) is a fuzzy number in the range $[0, \infty]$. The heading to a landmark $H(l)$ (or $H(t)$ to the target) is a fuzzy number with 
range $[0,2 \pi]$. For each of these, the imprecision $\left(I_{d}(l)\right.$ for distance, $I_{h}(l)$ for heading) is defined by taking the interval corresponding to the $70 \% \alpha$-cut of the fuzzy number (see Figure 2).

The imprecision of a landmark is computed by combining the imprecision in the heading and in the distance as follows. First, $I_{h}(l)$ is normalized by dividing by its maximum value of $2 \pi$. Second, $I_{d}(l)$ is normalized by applying the hyperbolic tangent function, which maps it into the $[0,1]$ interval. Finally, the two imprecisions are combined according to: $I(l)=\lambda \cdot \tanh \left(\beta \cdot I_{d}(l)\right)+(1-\lambda) \cdot \frac{I_{h}(l)}{2 \pi}$ where $\lambda$ weighs the relative importance of the two imprecisions, and $\beta$ controls how quickly the transformed $I_{d}$ approaches 1 . In our experiments, we set $\beta=1$ and $\lambda=0.2$.

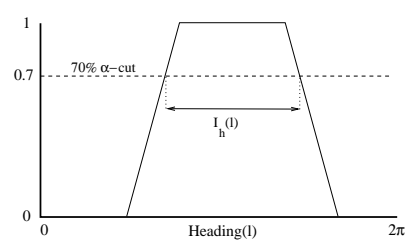

Figure 2: Computation of imprecision from a fuzzy number

We summarize the agent's knowledge of the landmarks in each sector by averaging the imprecision of the four most-precisely-known landmarks. The function Best : $N \times 2^{L} \rightarrow 2^{L}$ selects a subset, $B=\operatorname{Best}(n, L)$, of a group of landmarks, $L=\left\{l_{1}, \ldots, l_{m}\right\}$, such that $|B| \leq n \wedge$ $\forall \forall_{l \in B} \forall_{l^{\prime} \in L-B} I(l) \leq I\left(l^{\prime}\right)$. Having 4 landmarks in one sector is already very good, since only 3 landmarks are needed to use the beta-coefficient system network. Furthermore, we do not want these measures to be affected by bad landmarks when we have some that are good enough. That is why we use $\operatorname{Best}(4, L(s))$ when computing $\bar{I}(s)$.

\section{$5.2 \quad$ Features}

After computing these state variables, we discretize them to define a small number of features each of which takes on a small number of values. We employ the following features:

- Target Distance, $D(t)$, discretized to 5 intervals.

- Target Location Imprecision: measure of imprecision on the location of the target, $I(t)$, discretized to 7 intervals.
- Landmark Count: average number of landmarks over the six sectors, $\bar{C}=\frac{1}{6} \sum_{s=0}^{5} N(s)$, discretized to 4 intervals.

- Landmark Imprecision: average imprecision of landmarks' locations in each sector, $\bar{I}=$ $\frac{1}{6} \sum_{s=0}^{5} \bar{I}(s)$, discretized to 7 intervals.

This gives a total of 980 belief states.

\subsection{Actions}

Just as RL requires careful design of the state space to ensure that it is compact, it also requires careful design of the action set to ensure that it is small but also sufficient for the robot to achieve its goals. This is even more important when we are planning based on dynamic programming, then we must keep the state and action spaces small.

Physically, the robot is able to simultaneously perform two types of actions: moving and looking actions. Moving actions make the robot move in a given direction. Looking actions employ the camera to identify or track landmarks in the environment in specified sectors. The vision system can either search for new landmarks or re-acquire already-detected landmarks, but it is not able to do both things at the same time, because different image processing routines are required for each. In either case, however, the vision system returns the heading and distance to the landmarks it detects.

An additional constraint on the design of actions is that the vision system is most effective when the robot is moving in certain directions relative to the landmarks being observed.

Given these constraints, we have designed the following set of actions for the Learning Agent:

- Move Blind (MB): move toward the target (i.e., in the direction in which the target is believed to lie). Do not use the vision system.

- Move and Look for Landmarks (MLL): move toward the target. Point the camera in the sector that contains the fewest number of known landmarks, and look for new landmarks in this sector.

- Move Orthogonally to Target (MOT): move orthogonally to the direction of the target. Point the camera at the target and attempt to improve the precision of the heading and distance to the target. 
- Move and Verify Landmarks (MVL): move toward the target. Point the camera to the sector with the maximum imprecision, $\bar{I}$, and attempt to re-acquire known landmarks and measure their heading and distance more accurately.

- Move and Verify Target (MVT): move toward the target. Point the camera at the target and attempt to re-acquire it and measure its heading and distance more accurately.

These actions should affect the state variables as follows. All actions except MOT will make the distance to the target decrease. MB will make all imprecisions grow. MLL should increase the number of detected landmarks. MOT should reduce the imprecision about the target's location, while MVL should reduce the overall imprecision. MVT will also reduce the imprecision of the target's location, but not as much as MOT. All actions require that the heading to the target is known (at least approximately). The heading is choosen as the center of the fuzzy interval for $H(t)$. If the heading is completely unknown, the center of this interval will be $\pi$. This causes the robot "pace" back and forth, turning 180 degrees ( $\pi$ radians) each time an action is executed.

We have assigned an immediate reward to each action to reflect the load on the vision and the motion systems. The rewards are negative, because they are costs. MB is the cheapest action, since it does not use the camera. It has a reward of -1 . MVL and MVT produce a reward of -5 , since they make moderate demands on the vision system. MOT gives a reward of -6 , because it requires more motion in addition to the same image processing as MVL and MVT. Finally, MLL is the most expensive, with a reward of -10 , because it must do extensive image processing to search for new landmarks and verify that they are robust to changes in viewpoint.

The system receives a reward of 0 when it reaches the target. The RL objective is to maximize the total reward. In our case, it is equivalent to minimize the total cost of the actions taken to reach the target.

\section{Experimentation}

We employ the Webots ${ }^{1}$ simulator to perform our experiments. This simulator has a very high real-

\footnotetext{
${ }^{1}$ Webots simulator, www.cyberbotics.com
}

ism, adding noise to the robot movement and sensing. The simulated environment contains a set of landmarks, one of which is designated as the target. There is also a wall that surrounds the region in which the robot is navigating. The landmarks are the only objects in the environment. There are no obstacles, as obstacle avoidance is handled by the Pilot system. However, the robot can be blocked by the landmarks or by the wall. In each trial, the robot starts at a random location in this environment, and it has to reach the target. The trial terminates under three conditions: (a) if the robot reaches the target (and is confident that it has reached the target), (b) if the robot takes 500 steps without reaching the target, or (c) if the robot is blocked.

In order to see if the performance of the system improves after learning, we compare it with a handcoded policy. The hand-coded policy uses the same discretized features as the learning algorithm. If the robot is far from the target and there are few landmarks, it executes an MLL; if there are enough landmarks but they are highly imprecise, an MVL is executed; if the landmarks are good enough, an MOT is executed if the target's location has a high imprecision, and an MB is executed otherwise. If the robot is not far from the target but its location is highly imprecise, then an MVL or an MVT is executed, depending on whether the overall imprecision of landmarks is high or not; if the target's location has low imprecision, then if the robot is really near the target it executes an MVT, and an MB is executed if the distance is medium. This handcoded policy is not the same as the policy produced by the hand-coded bidding functions previously reported [14]. We have chosen this policy because it allows us to debug and test the Learning Agent separately from the rest of the multi-agent system.

The Learning Agent was trained for 2000 simulated trials. At regular intervals, the learned value function was tested by placing the robot in 99 randomly-chosen starting locations, running one trial from each location, and measuring the total reward, the total number of actions, and whether the robot succeeded in reaching the target position. The same set of 99 starting locations was employed in each testing period. The hand-coded policy was also evaluated on these 99 starting locations. Figure 3 shows that even after only 99 trials, the Learning Agent is already out-performing the hand-coded policy. After 2000 trials, the Learning Agent succeeds in reaching the target in 84 of the trials, com- 


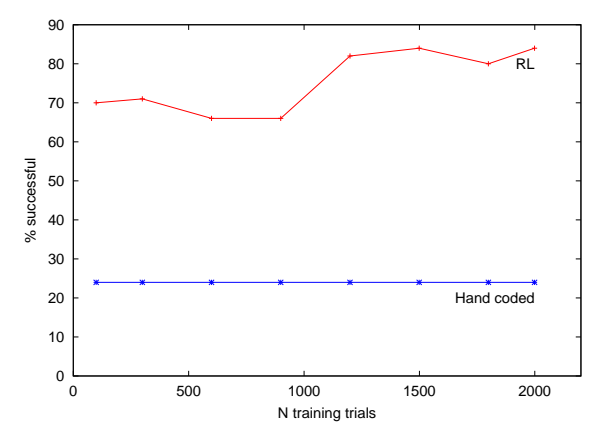

Figure 3: Number of successful test trials as a function of training steps.

pared to only 24 for the hand-coded policy.

We can also compute the average reward per trial, the number of actions per trial, and the number of actions of each type. Table 1 displays this information after 2000 training trials. Each value is averaged over five test runs. The only difference between test runs is the random number seed for the Webots simulator. We see that while the hand-coded policy receives an average of -858 units of reward, the learned policy only receives -336 units, which is a huge improvement. In addition, the Learning Agent on the average only requires 50 steps to terminate a trial (reach the goal, become blocked, or execute 500 steps) compared to 153 steps for the hand-coded policy. The Learning Agent never terminates because of the 500-step limit.

Looking at Table 1, we see that the Learning Agent has learned to perform fewer MOT and MVL actions and more MB, MVT, and MLL actions. Note particularly that the learning agent is executing an average of 11.4 MB actions per trial, compared to only 4.9 for the hand-coded policy. One of the goals of applying RL was to find a policy that freed the camera for use by the low-level obstacle avoidance routines, and this is exactly what has happened. On the other hand, we were surprised to see that the Learning Agent chooses to execute the most expensive action, MLL, so often (21.4 times per trial, compared to only 7.3 times per trial for the hand-coded policy). Evidentally, it has found that a mix of MLL and MB gives better reward than the combination of MVL and MOT that is produced by the hand-coded policy. The Learning Agent spends much more time looking for new landmarks and much less time verifying the direction and distance to known landmarks.

\section{Conclusion and future work}

The long-term goal of our research is to apply RL to automatically learn bidding functions in a multiagent system. In this paper, we have taken a step toward this goal by showing that RL can learn to select actions to resolve a complex tradeoff that arises in our multi-agent robot control system. The results of our experiments show that the Learning Agent achieves a higher rate of success than the hand-coded policy in the Navigation multi-agent system. Furthermore, the Learning Agent accomplishes this while performing many fewer actions and while performing a different mix of actions. This underlines the value of employing RL rather than attempting to resolve complex tradeoffs by hand-coding.

The next step will be to integrate the Learning Agent into the overall multi-agent system and compare the performance of the Learning Agent with the performance of the hand-coded bidding functions described in [14]. This will require that we find a way to scale the learned value function so that it provides appropriate bids within the MAS. We may also find that the Learning Agent should place a higher or lower cost on using the camera than we employed in the experiments described here. We can easily change the reward function to reflect increased or decreased costs and then retrain the Learning Agent.

Additional future work will also test the robustness of these results to changes in the set of features and the set of actions. The current sets of features and actions reflects lessons from three previous design iterations. A challenge was to design features and actions that were well-matched in the sense that the actions should frequently lead to a change in the feature values so that RL can detect progress in moving toward (or away from) the goal. If the actions operate at a finer grain than the features can represent, most actions appear to leave the state unchanged, and learning becomes impossible. This has been termed the "state-action deviation problem" [2]. It can be fixed by using more levels of discrete values for each feature, but this slows learning by requiring that the robot perform more interactions with the environment. Asada et al. [2] suggest another solution in which self-loops (cases where the state is unchanged) are ignored in the transition model. We plan to evaluate the suitability of this approach in future experiments.

In designing the current set of actions, we sought 
Table 1: Comparison of the Learning Agent (LA) and the hand-coded policy (HC) after 2000 training trials. RPT: Reward per trial. APT: Actions per trial

\begin{tabular}{rrrrrrrr} 
& RPT & APT & MB & MOT & MVT & MVL & MLL \\
\hline HC & -858 & 153.33 & 4.94 & 18.59 & 0.52 & 121.96 & 7.32 \\
LA & -336 & 49.95 & 11.41 & 6.52 & 5.61 & 4.97 & 21.43 \\
\hline
\end{tabular}

to minimize the number of actions to keep the branching factor of the exploratory search small. However, a consequence of this is that most of the actions have "built-in" decisions. For example, MLL always looks for new landmarks in the sector with the fewest landmarks, and this might not always be best. However, we did not want to introduce six different "look-for-landmarks" actions, one for each sector, because this would increase the branching factor and make the initial exploration very slow. Nonetheless, we would like to study whether it might be possible to introduce such finergrained actions after the Learning Agent has found a good initial policy. This might allow the learner to improve its performance further. In conclusion, $\mathrm{RL}$ is a promising method for tuning the behavior of agents in a multi-agent system.

\section{Acknowledgements}

This research has been supported by the Fulbright Joint Research Project and Plan Nacional Project DPI 2000-1352-C02-02. Dídac Busquets holds the CIRIT doctoral scholarship 2000FI-00191.

\section{References}

[1] R.C. Arkin. Motor schema-based mobile robot navigation. Int. J. Robotics research, 8(4):92-112, 1989.

[2] M. Asada, S. Noda, S. Tawaratsumida, and Koh Hosoda. Purposive behavior acquisition for a real robot by vision-based reinforcement learning. $M a$ chine Learning, 23:279-303, 1996.

[3] G. Bojadziev and M. Bojadziev. Fuzzy sets, fuzzy logic, applications, volume 5 of Advances in Fuzzy Systems. World Scientific, 1995.

[4] R. Brooks. A robust layered control system for a mobile robot. IEEE J. of Robotics and Automation, RA-2(1):14-23, 1986.

[5] A. R. Cassandra, L. P. Kaelbling, and M. L. Littman. Acting optimally in partially observable stochastic domains. In Proc. of the Twelfth National Conference on Artificial Intelligence, pages 1023-1028, Cambridge, MA, 1994. AAAI Press/MIT Press.

[6] M. Teresa Escrig and F. Toledo. Autonomous robot navigation using human spatial concepts. Int. J. of Intelligent Systems, 15:165-196, 2000.

[7] R. Liscano et al. Using a blackboard to integrate multiple activities and achieve strategic reasoning for mobile-robot navigation. IEEE Expert, 10(2):24-36, 1995.

[8] C. Isik and A.M. Meystel. Pilot level of a hierarchical controller for an unmanned mobile robot. IEEE J. Robotics and Automation, 4(3):242-255, 1988.

[9] T.S. Levitt and D.T. Lawton. Qualitative navigation for mobile robots. Artificial Intelligence Journal, 44:305-360, 1990.

[10] P. Maes. The dynamics of action selection. In Proc. of IJCAI'89, pages 991-997, 1989.

[11] A. W. Moore and C. G. Atkeson. Prioritized sweeping: Reinforcement learning with less data and less time. Machine Learning, 13:103, 1993.

[12] T.J. Prescott. Spatial representation for navigation in animats. Adaptive Behavior, 4(2):85-125, 1996.

[13] J. Rosenblatt. Damn: A distributed architecture for mobile navigation. In Proc. of the 1995 AAAI SpringSymposium on Lessons Learned from Implemented Software Architectures for Physical Agents. AAAI Press, March 1995.

[14] C. Sierra, R. López de Màntaras, and D. Busquets. Multiagent bidding mechanisms for robot qualitative navigation. In Intelligent Agents VII. Lectures Notes in Artificial Intelligencee (Proc. of ATAL2000), pages 198-212. Springer, Verlag, 2001.

[15] A. Stentz. The codger system for mobile robot navigation. In C.E. Thorpe, editor, Vision and Navigation, the Carnegie Mellon Navlab, pages 187201, Boston, 1990. Kluwer Academic Pub. 